\title{
Eficiência Técnica de Aeroportos: uma abordagem focada na infraestrutura aeroportuária - Lado Ar
}

\author{
Antonio Márcio Ferreira Crespo \\ Air Navigation Commission - International Civil Aviation Organization- Montreal - \\ Canada \\ acrespoeicao.int
}

\begin{abstract}
Resumo. As sedes de grandes eventos esportivos se tornam centros turísticos de notória atratividade, implicando grande incremento de demanda por deslocamentos aéreos. Neste contexto, o presente trabalho objetivou analisar de que forma a configuração da infraestrutura aeroportuária condicionou o desempenho de dezessete aeroportos que apoiarão as sedes da Copa 2014. Os dados foram processados segundo a abordagem de análise multivariável denominada Análise de Envoltória de Dados. Os resultados obtidos indicam que alguns aeroportos podem ter ganhos de capacidade apenas com a otimização de procedimentos operacionais; outros, contudo, carecem de intervenções na infraestrutura para obter tal incremento.
\end{abstract}

Abstract.: Huge sports events host cities become centers of great tourist attraction, implying large increase in demand for air travel. In this context, the present study aimed to analyze the role of airport infrastructure as conditioning factor to the capacity of seventeen airports that support the 2014 World Cup. The data were processed according to multivariable analysis approach called Data Envelopment Analysis (DEA), by using the tool called Integrated Decision Support System (SIAD). The results indicate that some airports may have capacity gains by optimizing operating procedures; others, however, may need infrastructure interventions to obtain such gain.

\section{Introdução}

Em 31 de maio de 2009 foram definidas as cidades-sedes: Belo Horizonte, Brasília, Cuiabá, Curitiba, Fortaleza, Manaus, Natal, Porto Alegre, Recife, Rio de Janeiro, Salvador e São Paulo. A análise de eventos anteriores, tais como a Copa do Mundo 2010 realizada na África do Sul, indica que o país-sede se torna extremamente atrativo sob o ponto de vista da atividade turística nacional e internacional.

Estudo coordenado pela Fundação Getúlio Vargas, a pedido do Ministério do Turismo [Ernst \& Young; FGV Projetos, 2010], aponta que o evento Copa do Mundo 2014 pode trazer ao Brasil 600.000 turistas estrangeiros que deverão realizar cerca de 2 milhões de deslocamentos no país, preponderantemente por via aérea. Por sua vez, estima-se que 3 milhões de Brasileiros realizarão algo em torno de 6 milhões de deslocamentos durante a Copa. Tal projeção aponta para uma elevada demanda específica de tráfego aéreo (demanda aérea), a qual deverá ser analisada e gerenciada em conjunto com o crescimento de volume de tráfego verificado no país.

Ao considerar o contexto ora apresentado, verifica-se a importância do desenvolvimento de estudos que tenham como objeto o desempenho e a eficiência dos 
aeroportos que, de forma direta ou indireta, apoiarão a realização de tão importante evento.

Neste contexto, o presente trabalho objetivou analisar de que forma a configuração da infraestrutura aeroportuária (Lado Ar) condicionou o desempenho operacional, medido em termos de capacidade horária (pousos e decolagens) [BRASIL, 2009], dos dezessete aeroportos que apoiarão, de forma direta, as cidades-sede da Copa 2014.

A análise em tela focou dois componentes dessa infraestrutura, quais sejam, as pistas de pouso e as pistas de taxi para saída rápida existentes, considerando a influência destas sobre a capacidade horária dos sistemas de pistas (valores em vigor). Para tanto, empregou-se a abordagem de análise multivariável denominada Análise de Envoltória de Dados (DEA - Data Envelopment Analysis), derivando-se o indicador Eficiência Técnica.

\section{Revisão da Literatura}

O Sistema de Aviação Civil é composto basicamente pela Aviação Civil, pela Infraestrutura Aeronáutica e pela Infraestrutura Aeroportuária, nos termos do que preconiza a Lei 7.565, de 19 de dezembro de 1986 - Código Brasileiro de Aeronáutica (CBA) [BRASIL, 1986].

\subsection{A Aviação Civil}

O impacto da indústria de transporte aéreo comercial sobre a economia mundial é relevante, contribuindo com mais de US \$2,6 trilhões, o equivalente a oito por cento do produto interno bruto mundial do ano de 2008 [Horonjeff et al., 2010].

Conforme definido pelo CBA [BRASIL, 1986], o sistema aeroportuário é constituído pelo conjunto de aeródromos brasileiros, com todas as pistas de pouso, pistas de táxi, pátio de estacionamento de aeronave, terminal de carga aérea, terminal de passageiros e os respectivos serviços. Excluindo-se os serviços, os demais itens listados constituem a infraestrutura aeroportuária. Suportado por Tió (2010), ressalta-se que os principais componentes da Infraestrutura Aeroportuária são agrupados e classificados por: Componentes do Lado Ar e Componentes do Lado Terra.

$\mathrm{O}$ "Lado Ar" refere-se às instalações aeroportuárias associadas com o movimento de aeronaves, utilizadas principalmente para pouso e decolagem, por exemplo, pistas, taxiways e instalações ATC. Alguns dos principais componentes do Lado Ar são: a) Pista de Pouso e Decolagem, b) Pista de Taxi, c) Pista de Taxi de Saída Rápida. O "Lado Terra" refere-se às estruturas de processamento de passageiros e de processamento e armazenamento de carga, além dos pátios de manobra e estacionamento de aeronaves

\subsection{Produtividade e Eficiência em Aeroportos}

Apoiando-se em Liebert e Niemeier (2010), infere-se que a produtividade de um aeroporto, bem como a de qualquer um de seus componentes, pode ser simplesmente definida como a proporção entre resultados/desempenho (saídas-outputs) por recursos/meios disponíveis (entradas-inputs). A divisão de uma saída por uma entrada (por exemplo, a produtividade do trabalho: passageiros por empregado) dá uma indicação da produtividade parcial do referido aeroporto. Ao se agregar todos os fatores 
(entradas e saídas) inerentes à atividade aeroportuária, obtem-se uma medida geral conhecida na literatura como Produtividade Total dos Fatores (Total Factor Productivity - TFP).

A eficiência é definida pela comparação entre determinada produtividade e a produtividade máxima observada, desde que as entradas/saídas sejam equivalentes. A eficiência também pode ser estabelecida a partir de uma relação ótima entre resultados/desempenho e recursos/meios disponíveis.

A avaliação de eficiência, de forma geral, pode ser viabilizada mediante a aplicação de três métodos quantitativos, a saber: a) abordagens não paramétricas baseadas em indexação numérica para medir TFP; b) abordagens paramétricas tais como a Análise de Fronteira Estocástica (Stochastic Frontier Analysis - SFA); e c) abordagens não paramétricas baseadas em programação linear, com destaque para a Análise Envoltória de Dados (Data Envelopment Analysis - DEA).

\section{Metodologia}

A primeira etapa do trabalho desenvolvido consistiu da definição e estudo dos eixos teóricos que balizaram a coleta e o processamento dos dados e informações de interesse para a pesquisa. Em seguida, passou-se para a busca de informações junto ao CGNA, compilando-se o indicador operacional capacidade dos sistemas de pistas dos aeroportos (em vigor) que atendem, de forma direta, às doze cidades-sede da Copa do Mundo 2014. Os indicadores de capacidade calculados pelo CGNA são apresentados na Seção 3 deste artigo, e o Quadro 1 lista os aeroportos estudados.

Quadro 1. Aeroportos de apoio direto à Copa do Mundo 2014.

\begin{tabular}{|c|c|c|}
\hline CIDADE & $\begin{array}{c}\text { CÓDIGO } \\
\text { ICAO }\end{array}$ & NOME \\
\hline \multirow{2}{*}{ Belo Horizonte } & SBBH & Aeroporto Carlos Drumond de Andrade \\
\cline { 2 - 3 } & SBCF & Aeroporto Internacional Tancredo Neves \\
\hline Brasília & SBBR & Aeroporto Internacional Presidente Juscelino Kubitschek \\
\hline Campinas & SBKP & Aeroporto Internacional de Viracopos \\
\hline Cuiabá & SBCY & Aeroporto Internacional Marechal Rondon \\
\hline Curitiba & SBCT & Aeroporto Internacional Afonso Pena \\
\hline Fortaleza & SBFZ & Aeroporto internacional Pinto Martins \\
\hline Goiânia & SBGO & Aeroporto Internacional Santa Genoveva \\
\hline Manaus & SBEG & Aeroporto Internacional Eduardo Gomes \\
\hline Natal & SBNT & Aeroporto Internacional Augusto Severo \\
\hline Porto Alegre & SBPA & Aeroporto Internacional Salgado Filho \\
\hline Recife & SBRF & Aeroporto Internacional dos Guararapes \\
\hline \multirow{2}{*}{ Rio de Janeiro } & SBGL & Aeroporto Internacional Antonio Carlos Jobim \\
\cline { 2 - 3 } & SBRJ & Aeroporto Santos Dumont \\
\hline Salvador & SBSV & Aeroporto Internacional Deputado Luís Eduardo Magalhães \\
\hline \multirow{2}{*}{ São Paulo } & SBGR & Aeroporto Internacional Governador André Franco Montoro \\
\cline { 2 - 3 } & SBSP & Aeroporto de Congonhas \\
\hline
\end{tabular}

Num terceiro momento, procedeu-se a análise da infraestrutura aeroportuária inerente aos aeroportos listados. Mediante o estudo das Cartas de Aeródromo (ADC) disponibilizadas pelo DECEA [Brasil, 2013], foi possível deduzir dois indicadores da infraestrutura aeroportuária existente, a saber: quantidade de pistas de pouso e quantidade de pistas de taxi de saída rápida. 
Sequencialmente, os dados e informações obtidos (capacidade dos sistemas de pistas de pouso, quantidade de pistas de pouso e quantidade de pistas de taxi de saída rápida) foram utilizados para produzir indicadores de eficiência.

Dentre as diversas abordagens voltadas para a análise de eficiência, optou-se pelo emprego do método multivariável Análise de Envoltória de Dados (DEA - Data Envelopment Analysis), em função das suas características e vantagens.

A abordagem DEA, fundamentada pela teoria de resolução de problemas de programação linear com múltiplas entradas (insumos - inputs) e saídas (produtos outputs), é utilizada para avaliar a eficiência dos aeroportos, considerados como Unidades de Tomada de Decisão (Decision Making Units - DMU) [Charnes et al., 1978].

O modelo DEA CRS (ou DEA CCR) mede a eficiência técnica global, abrangendo a eficiência técnica pura e a eficiência de escala. O modelo DEA VRS (ou DEA BCC), por sua vez, mede somente a eficiência técnica pura. Assim sendo, a pesquisa foi desenvolvida com o suporte da abordagem DEA VRS. Com isso buscou-se neutralizar os efeitos de escala decorrentes da discrepância entre os aeroportos, em termos de dimensão, quantidade de movimento anual, mix de aeronaves, tipo de voo (nacional / internacional), bem como quaisquer outras características que pudessem redundar em ganhos de escala.

Os dados e informações coletados, portanto, foram processados mediante a utilização da ferramenta denominada Sistema Integrado de Apoio à Decisão (SIAD) [Angulo-Meza et al., 2005], software livre amplamente utilizado para análise de eficiência segundo a abordagem DEA. Os critérios aplicados ao SIAD estão explicitados no Quadro 2.

Quadro 2. Critérios utilizados na ferramenta SIAD.

\begin{tabular}{|l|l|}
\hline Método & DEA VRS \\
\hline Orientação & Output / Input \\
\hline Inputs & $\begin{array}{l}\text { Quantidade de pistas de pouso e Quantidade de } \\
\text { pistas de taxi para saída rápida }\end{array}$ \\
\hline Output & Capacidade horária do sistema de pistas \\
\hline Eficiência & Composta \\
\hline
\end{tabular}

\section{Resultados e Discussão}

Conforme destacado anteriormente, os dados e informações obtidos foram processados mediante a utilização do software SIAD. A Tabela 1 quantifica os componentes da infraestrutura aeroportuária analisados (inputs para o SIAD) e apresenta a capacidade horária dos sistemas de pistas nos aeroportos estudados (output para o SIAD), no ano de 2012.

Por oportuno, cabe ressaltar que as cabeceiras 02L / 20R de SBRJ e 12 / 30 de SBNT foram desconsideradas, posto que as mesmas não são utilizadas na operação comum diária. 
Tabela 1. Indicadores de infraestrutura aeroportuária e capacidade horária dos sistemas de pistas - valores em vigor.

\begin{tabular}{cccc|cccc}
\hline DMU & \multicolumn{2}{c}{ Input } & Output & DMU & \multicolumn{2}{c}{ Input } & Output \\
\hline Aeroporto & RWY $^{\mathbf{a}}$ & TWY $^{\mathbf{a}}$ & Mov/H $^{\mathbf{b}}$ & Aeroporto & RWY $^{\mathbf{a}}$ & TWY $^{\mathbf{a}}$ & Mov/ $^{\mathbf{b}}$ \\
\hline SBGR & 4 & 4 & 47 & SBCT & 4 & 1 & 24 \\
SBSP & 4 & 1 & 36 & SBRF & 2 & 4 & 29 \\
SBBR & 4 & 5 & 44 & SBFZ & 2 & 2 & 27 \\
SBRJ & 2 & 1 & 29 & SBGO & 2 & 0 & 26 \\
SBGL & 4 & 7 & 40 & SBBH & 2 & 1 & 25 \\
SBSV & 4 & 1 & 25 & SBCY & 2 & 0 & 22 \\
SBCF & 2 & 1 & 29 & SBEG & 2 & 0 & 25 \\
SBKP & 2 & 3 & 28 & SBNT & 4 & 1 & 27 \\
SBPA & 2 & 0 & 24 & - & - & - & - \\
\hline
\end{tabular}

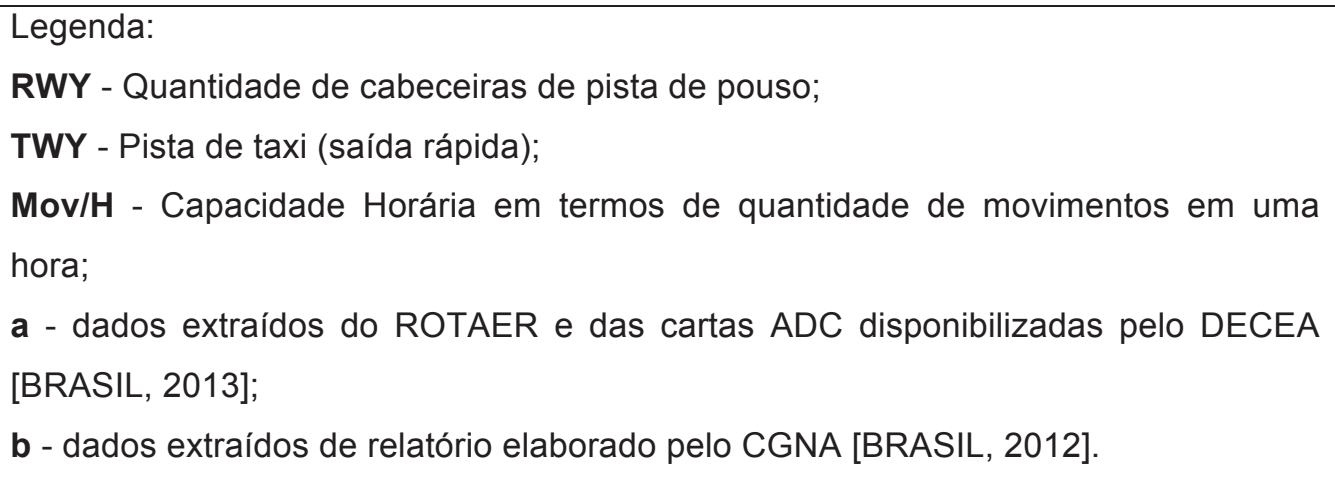

À eficiência definida a partir do output capacidade horária pode ser atribuído um enfoque eminentemente operacional. Na medida em que o Modelo DEA VRS permite estabelecer índices de eficiência técnica, tendo como entrada elementos quantitativos de infraestrutura comuns aos aeroportos analisados, torna-se possível estabelecer uma indexação que reflete a forma como a operação transcorre no dia-a-dia.

O dados constantes da Tabela 1 foram processados pela ferramenta SIAD. A Tabela 2 apresenta os resultados obtidos, ou seja, a eficiência técnica dos 17 aeroportos estudados, definida em função dos inputs quantidade de cabeceiras de pista de pouso e quantidade de pistas de taxi para saída rápida, bem como do output capacidade horária. 
Tabela 2. Eficiência do conjunto de pistas segundo o Modelo DEA BCC orientado a output sem benchmark.

\begin{tabular}{lccc}
\hline DMU & $\begin{array}{c}\text { Eficiência } \\
\text { Padrão }\end{array}$ & $\begin{array}{c}\text { Eficiência } \\
\text { Invertida }\end{array}$ & $\begin{array}{c}\text { Eficiência } \\
\text { Composta }\end{array}$ \\
\hline SBSP & 1 & 0,666667 & 0,666667 \\
SBGR & 1 & 0,680851 & 0,659574 \\
SBRJ & 1 & 0,818965 & 0,590517 \\
SBCF & 1 & 0,818965 & 0,590517 \\
SBGO & 1 & 0,846154 & 0,576923 \\
SBBR & 0,93617 & 0,787879 & 0,574146 \\
SBEG & 0,961538 & 0,88 & 0,540769 \\
SBPA & 0,923077 & 0,916667 & 0,503205 \\
SBRF & 1 & 1 & 0,5 \\
SBKP & 0,965517 & 0,973214 & 0,496151 \\
SBFZ & 0,931034 & 0,944444 & 0,493295 \\
SBBH & 0,862069 & 0,95 & 0,456035 \\
SBNT & 0,75 & 0,888889 & 0,430556 \\
SBGL & 0,851064 & 1 & 0,425532 \\
SBCY & 0,846154 & 1 & 0,423077 \\
SBSV & 0,694444 & 0,96 & 0,367222 \\
SBCT & 0,666667 & 1 & 0,333333 \\
\hline
\end{tabular}

Com base na Tabela 2, é possível listar os aeroportos que se destacam positivamente pela produtividade relativa (SBSP, SBGR, SBRJ, SBCF, SBGO e SBRF) na exploração da infraestrutura existente, na medida em que apresentam Eficiência Padrão igual a 1. Da mesma forma, listam-se aeroportos que se destacam negativamente neste critério (SBRF, SBGL, SBCY e SBCT), posto que apresentam Eficiência Invertida (ineficiência) igual a 1 .

Os dados acima sinalizam que SBRF é uma DMU "falsa eficiente" típica. De modo semelhante, outros aeroportos apresentam elevada eficiência padrão (exploram bem seus pontos fortes), mas também apresentam destacada eficiência invertida (ineficiência - são muito impactados pelos seus pontos fracos), como por exemplo: SBKP, SBFZ e SBPA. Desta feita, conforme discutido previamente, a Eficiência Composta é o parâmetro utilizado neste trabalho para indexar os aeroportos.

Convém destacar que a Tabela 2 permite classificar os aeroportos estudados com base nas práticas operacionais adotadas no país. Entretanto, o modelo DEA possibilita a realização de uma análise comparativa referenciada por práticas operacionais adotadas em localidades de referência. Para tanto, basta alimentar o modelo com os indicadores observados nestas localidades, que serão tomados como benchmark.

Com o objetivo de estabelecer tal análise, foram escolhidos como benchmark os seguintes aeroportos: Aeroporto Internacional de Heathrow (EGLL) e Aeroporto Internacional de Gatwick (EGKK). A inclusão dos aeroportos britânicos como benchmark permite estabelecer uma comparação mais valiosa entre os padrões operacionais de utilização da infraestrutura, na medida em que tais aeroportos são referência mundial neste quesito e apresentam infraestrutura semelhante à que se observa no país. Tal assertiva se justifica pelos seguintes fatos: a) Heathrow (EGLL) é o 
aeroporto com duas pistas de maior movimento no mundo; b) Gatwick (EGKK) é o aeroporto com uma pista de maior movimento no mundo [Airport Council International, 2013].

A Tabela 3 apresenta os valores obtidos para a Eficiência Composta segundo as duas condições: sem benchmark e com benchmark.

Tabela 3. Análise comparativa de eficiências compostas.

\begin{tabular}{llcc}
\hline & DMU & $\begin{array}{c}\text { Eficiência Composta } \\
\text { RWY+TWY Benchmark }\end{array}$ & $\begin{array}{c}\text { Eficiência Composta } \\
\text { RWY+TWY }\end{array}$ \\
\hline- & EGKK & 0,669697 & - \\
1 & SBSP & 0,666667 & $0,666667(1)$ \\
2 & SBGR & 0,655355 & $0,659574(2)$ \\
3 & SBGO & 0,576923 & $0,576923(5)$ \\
4 & SBRJ & 0,560788 & $0,590517(3)$ \\
5 & SBCF & 0,560788 & $0,590517(4)$ \\
6 & SBEG & 0,540769 & $0,540769(7)$ \\
7 & SBBR & 0,535748 & $0,574146(6)$ \\
8 & SBPA & 0,503205 & $0,503205(8)$ \\
- & EGLL & 0,5 & - \\
9 & SBNT & 0,430556 & $0,430556(13)$ \\
10 & SBBH & 0,430405 & $0,456035(12)$ \\
11 & SBCY & 0,423077 & $0,423077(15)$ \\
12 & SBFZ & 0,406283 & $0,493295(11)$ \\
13 & SBSV & 0,367222 & $0,367222(16)$ \\
14 & SBKP & 0,359072 & $0,496151(10)$ \\
15 & SBGL & 0,343137 & $0,425532(14)$ \\
16 & SBCT & 0,333333 & $0,333333(17)$ \\
17 & SBRF & 0,319853 & $0,5(9)$ \\
\hline
\end{tabular}

Conforme pode ser observado na Tabela 3, a inclusão dos aeroportos britânicos fez com os valores de eficiência dos aeroportos brasileiros se reduzissem ou, no máximo, permanecessem inalterados. Paralelamente às eficiências calculadas, a ferramenta SIAD disponibiliza projeções que tornam possível inferir sobre a influência dos indicadores de infraestrutura sobre a capacidade, bem como sobre a adequabilidade dos procedimentos operacionais empregados para explorar a infraestrutura. O software em tela apresenta valores não inteiros, os quais devem ser interpretados como indicadores de tendência.

A Tabela 4, obtida mediante a utilização dos modelos DEA VRS orientados a output e input, ambos com benchmark, consolida de forma clara o objetivo proposto pela pesquisa, na medida em que evidencia a relação entre os componentes da infraestrutura aeroportuária e a capacidade dos sistemas de pista dos 17 aeroportos que apoiarão a Copa do Mundo de 2014. 
Tabela 4. Análise comparativa de valores alvo.

\begin{tabular}{c|cccc|c|c|c}
\hline \multirow{2}{*}{ DMU } & \multicolumn{2}{|c|}{ Minimização de Inputs } & & \multicolumn{3}{|c}{ Maximização de Outputs } \\
& RWY & TWY & $\begin{array}{c}\text { Capacida } \\
\text { de Atual }\end{array}$ & RWY & TWY & $\begin{array}{c}\text { Capacidade } \\
\text { Projetável }\end{array}$ \\
\hline SBGR & $2,84(4)$ & $3,89(4)$ & 47 & $4(2,8)$ & 4 & 47,4 \\
SBSP & $4(4)$ & $1(1)$ & 36 & 4 & 1 & 36 \\
SBBR & $2,68(4)$ & $3,35(5)$ & 44 & $4(2,4)$ & 5 & 51,2 \\
SBRJ & $2(2)$ & $0,62(1)$ & 29 & 2 & 1 & 30,83 \\
SBGL & $2(4)$ & $3,5(7)$ & 40 & $4(2,28)$ & 7 & 58,28 \\
SBSV & $2(4)$ & $0(1)$ & 26 & 4 & 1 & 36 \\
SBCF & $2(2)$ & $0,62(1)$ & 29 & 2 & 1 & 30,83 \\
SBKP & $2(2)$ & $0,41(3)$ & 28 & 2 & 3 & 40,5 \\
SBPA & $2(2)$ & $0(0)$ & 24 & 2 & 0 & 26 \\
SBCT & $2(2)$ & $0(1)$ & $24(26)$ & 4 & 1 & 36 \\
SBRF & $2(2)$ & $3,11(4)$ & 29 & 2 & 4 & 45,33 \\
SBFZ & $2(2)$ & $0,20(2)$ & 27 & 2 & 2 & 35,66 \\
SBGO & $2(2)$ & $0(0)$ & 26 & 2 & 0 & 26 \\
SBBH & $2(2)$ & $0(1)$ & 25 & 2 & 1 & 30,83 \\
SBCY & $2(2)$ & $0(0)$ & 22 & 2 & 0 & 26 \\
SBEG & $2(2)$ & $0(0)$ & 25 & 2 & 0 & 26 \\
SBNT & $2(2)$ & $0,20(1)$ & 27 & 4 & 1 & 36 \\
\hline
\end{tabular}

O grupo classificado como "Minimização de Inputs" agrega os indicadores de infraestrutura suficientes (valores mínimos) para manter as capacidades atuais. Nas colunas "RWY" e "TWY" deste grupo, os números entre parênteses representam os valores inerentes à infraestrutura existente. Na coluna "Capacidade Atual", os números entre parênteses indicam que é possível incremento de capacidade mesmo empregando menos infraestrutura, caso ilustrado pelo aeroporto SBCT.

O grupo classificado como "Maximização de Outputs" agrega os valores de capacidade que podem ser alcançados, mediante a otimização de procedimentos operacionais, empregando a infraestrutura existente. Na coluna "RWY" deste grupo, os números entre parênteses indicam o quanto da infraestrutura existente pode ser efetivamente explorada, decorrendo que fatores exógenos podem estar restringindo a operação do aeroporto.

Conforme pode ser verificado, os valores apresentados acima representam os indicadores mínimos de infraestrutura aeroportuária requeridos para manter as capacidades atuais (Minimização de Inputs), fornecendo indícios da forma como a referida infraestrutura está sendo explorada. Em adição, a mesma tabela apresenta os valores de capacidade que podem ser alcançados (Maximização de Outputs), mantendose os indicadores atuais, mediante a implementação de procedimentos otimizados, tendo como referência as práticas operacionais dos aeroportos de referência (EGLL e EGKK).

A partir das informações consolidadas na Tabela 4, pode-se definir 3 grupos de aeroportos, conforme apresentado no quadro a seguir. 
Quadro 3. Grupos de aeroportos segundo o nível de utilização da infraestrutura.

\begin{tabular}{|c|c|c|}
\hline Grupo & Caracterização & Aeroportos \\
\hline \multirow{3}{*}{ A } & $\begin{array}{c}\text { Infraestrutura explorada em sua plenitude. Procedimentos } \\
\text { operacionais plenamente ajustados à infraestrutura existente. }\end{array}$ & SBGR \\
& SBSP \\
& SBGO \\
\hline \multirow{3}{*}{ B } & Infraestrutura explorada de forma adequada. Procedimentos & SBRJ \\
& operacionais ajustados à infraestrutura, cabendo ajustes que & SBPA \\
& promovam pequenos ganhos de capacidade. & SBCY \\
& & SBEG \\
\hline \multirow{4}{*}{ C } & SBBR \\
& Infraestrutura subutilizada. Procedimentos operacionais carentes & SBGL \\
& Se aprimoramento. Possibilidade de interferência de fatores & SBKP \\
& SBCT \\
& exógenos restringindo a operação. & SBRF \\
& & SBFZ \\
& & SBBH \\
& & SBNT \\
\hline
\end{tabular}

As informações consolidadas na Tabela 4 e no Quadro 3 permitem a colocação de algumas inferências, relacionadas ao apoio que os aeroportos analisados podem oferecer para a realização da Copa do Mundo 2014. Conforme levantado no início deste trabalho, a realização de eventos desta natureza pressupõe incremento de demanda e pressão sobre a infraestrutura aeroportuária. Neste sentido, são apresentadas as inferências explicitadas abaixo:

a) com relação aos aeroportos que constituem o Grupo $\mathrm{A}$, não se pode esperar ganhos de capacidade sem intervenções que ampliem a infraestrutura existente; ou seja, ganhos de capacidade necessariamente dependem da construção de novas pistas de pouso e novas pistas de taxi de saída rápida;

b) com relação aos aeroportos do Grupo B, ganhos de capacidade significativos somente serão alcançados mediante intervenções que ampliem a infraestrutura existente; contudo, pequenos ganhos de capacidade podem ser derivados da otimização de procedimentos operacionais; e

c) com relação aos aeroportos do Grupo C, ganhos de capacidade significativos podem ser obtidos mediante a otimização de procedimentos operacionais.

Às considerações supracitadas cabe associar algumas ressalvas, decorrentes das limitações impostas à pesquisa realizada. Primeiramente, dado o caráter determinístico da abordagem DEA, o trabalho não agregou qualquer discussão sobre a incerteza inerente aos valores de eficiência obtidos. Assim, todos os valores de eficiência apresentados devem ser compreendidos como estimativas de eficiência.

Da mesma forma, a restrição do escopo, limitado a dois itens da infraestrutura aeroportuária, pressupõe que todos os outros elos da cadeia produtiva (demais componentes da infraestrutura) não apresentam quaisquer restrições de capacidade, o que provavelmente não condiz com a realizada. Desta feita, esta pesquisa pode ser valiosamente complementada pela paulatina inclusão dos demais componentes de infraestrutura. 


\section{Conclusão}

O presente trabalho levantou a eficiência técnica dos dezessete aeroportos brasileiros que, de forma direta, apoiarão a realização da Copa do Mundo de Futebol 2014. O foco da análise foi direcionado para dois componentes da infraestrutura aeroportuária (Lado Ar), a saber: pistas de pouso e pistas de taxi para saída rápida. Com isso, foi possível analisar de que forma a configuração da infraestrutura aeroportuária condicionou o desempenho operacional desses aeroportos, medido em termos de capacidade horária (pousos e decolagens).

Os indicadores obtidos foram processados com a finalidade de se derivar as estimativas de eficiência técnica inerentes aos aeroportos estudados. Para tanto foi utilizada a ferramenta computacional denominada Sistema Integrado de Apoio à Decisão (SIAD), a qual viabilizou a aplicação da abordagem Análise de Envoltória de Dados (DEA - Data Envelopment Analysis).

Os resultados da pesquisa sinalizaram que os aeroportos estudados apresentam três padrões de utilização da infraestrutura existente. O primeiro grupo se caracteriza pela plena utilização dos meios físicos existentes. Com relação a este grupo, ressaltou-se que ganhos de capacidade somente poderão ser obtidos mediante intervenções que expandam a infraestrutura aeroportuária da localidade.

O segundo grupo de aeroportos abarca aqueles que exploram de forma satisfatória a infraestrutura disponível; contudo, a otimização de procedimentos operacionais pode promover algum ganho de capacidade.

O terceiro grupo agrega os aeroportos que exploram a infraestrutura existente ineficientemente; assim sendo, espera-se que a otimização de procedimentos operacionais redunde em ganhos de capacidade relevantes.

Neste momento cabe ressaltar que os resultados obtidos devem ser submetidos a depurações posteriores, na medida em que a metodologia DEA produz estimativas de eficiência desprovidas de quaisquer tratamento quanto a incertezas. Da mesma forma, sugere-se que trabalhos futuros incorporem mais componentes da infraestrutura aeroportuária, tais como o pátio de estacionamento.

\section{REFERÊNCIAS}

Airport Council International. (2013). Preliminary 2012 World Airport Traffic and Rankings. Media Release. Montreal.

Angulo Meza, L., Biondi Neto, L., Soares de Mello, J.C.C.B., Gomes, E.G. (2005) "ISYDS - Integrated System for Decision Support (SIAD - Sistema Integrado de Apoio à Decisão): a software package for data envelopment analysis model", Pesquisa Operacional, v. 25, n. 3, p. 493-503.

Brasil. Comando da Aeronáutica. Centro de Gerenciamento da Navegação Aérea. (2012). Capacidade do Sistema de Pistas e Infraestrutura Disponível dos Aeroportos Selecionados para a Copa do Mundo de 2014. Rio de Janeiro, RJ.

Brasil. Comando da Aeronáutica. Departamento de Controle do Espaço Aéreo. (2009) MCA 100-14: capacidade do sistema de pistas. Rio de Janeiro, RJ. 
Brasil. Comando da Aeronáutica. Departamento de Controle do Espaço Aéreo. (2013) Serviço de Informação Aeronáutica, http://www.aisweb.aer.mil.br, Abril.

Brasil. Lei $\mathrm{n}^{\mathrm{o}}$ 7.565, de 19 de dezembro de 1986. (1986). Código Brasileiro de Aeronáutica. Diário Oficial da União, Brasília, DF, 20 dez. 1986.

Charnes, A., Cooper, W.W., Rhodes, E. (1978) "Measuring the Efficiency of Decision Making Units", European Journal of Operational Research, v. 2, n. 6, p. 429-444.

Ernst \& Young, FGV Projetos. (2010). Brasil Sustentável: impactos Socioeconômicos da Copa do Mundo 2014. Departamento de Comunicação e Gestão da Marca Ernst \& Young Brasil.

Horonjeff, R.; Mckelvey, F. X.; Sproule, W. J.; Young, S. B. (2010). Planning and Design of Airports. 5th Ed. New York: Mcgraw-Hill.

Liebert, V., Niemeier, H. (2010) "Benchmarking of Airports: a critical assessment", In: Proceedings of WCTR World Conference, Lisbon.

Tió M. V., Sevillano R. M. (2010). Study of Airport Capacity vs. Efficiency SESAR Challenges. Barcelona: INDRA - Advanced Logistic Groups. 